\title{
Phase shifter based on an ultrathin superconducting bilayer with a through-hole for a superconducting device
}

Hiroshi Ishizu ${ }^{1,2, *}$, Hirotake Yamamori ${ }^{1}$, Shunichi Arisawa ${ }^{3}, K^{2}$ azuyasu Tokiwa ${ }^{2}$, Yasumoto Tanaka ${ }^{1}$

1 National Institute of Advanced Industrial Science and Technology (AIST), Tsukuba, Ibaraki 305-8568, Japan

2 Faculty of Industrial Science and Technology, Tokyo University of Science, Katsushika, Tokyo 125-8585, Japan

${ }^{3}$ National Institute for Materials Science, Tsukuba, Ibaraki 305-0047, Japan

*Author to whom correspondence should be addressed: 8120506@ed.tus.ac.jp, https://orcid.org/0000-0002-9871-775X.

Other author ORCHID numbers:

Yamamori Hirotake (https://orcid.org/0000-0002-7296-711X)

Arisawa Shunichi (https://orcid.org/0000-0001-8155-9401)

Tokiwa Kazuyasu (https://orcid.org/0000-0003-3543-1786)

Tanaka Yasumoto (http://orcid.org/0000-0001-6421-2799, https://orcid.org/00000003-3543-178)

\section{Abstract}

Applying a radian phase shift other than $2 \pi$ is a key issue for superconducting circuits, such as flux qubits. The magnetic flux is useful 
when generating a phase shift. However, a quantized magnetic flux accompanying a trapped vortex in a superconductor does not possess a phase shifter function. The magnetic flux generated by an external field generates noise. In this study, we propose a phase bias system that does not require an external field during operation. We confirm the phase shift of a direct current superconducting interference device (SQUID) placed on an ultrathin superconducting $\mathrm{Nb}$ bilayer with a through-hole by cooling it to a temperature below the superconducting transition temperature with an external field. Although the cause of the phase shift in our system is unclear, we believe that it may be caused by a fractional quantum in the bilayer. When the SQUID is replaced by a qubit, the phase shift can be applied to a phase bias.

Keywords: Phase shifter, superconducting bilayer, SQUID, fractional quantum, $i$-soliton, multiband/multicomponent superconductor.

\section{Introduction}

Since 1971, multilayers of ultrathin superconducting sheets have been studied as two-dimensional superconductors [1] and as models of layered superconducting materials [2-5], such as cuprate- and Fe-based superconductors $[6,7]$. An ultrathin superconducting bilayer system is the simplest multilayer system [8]. Moreover, it can be considered as a model system of two-band/multicomponent superconductors [9, 10]. Phase difference solitons between components ( $i$-solitons) in 
multicomponent superconductors, which are not found in conventional superconductors, have been discussed and have been shown to increase the possibility of fractional flux quantum generation [10-13].

Hence, we developed a new ultrathin bilayer system to investigate these new topological objects found in multicomponent superconductors. In 2018, we confirmed the fractional quantization of magnetic flux in the ultrathin bilayer system with the use of a scanning magnetometer [14]. The scanning magnetometer provides a precise measurement of the magnetic flux distribution [15]. However, it requires the presence of a person throughout the measurement process, and it is difficult to measure the magnetic field dependence at low temperatures automatically. To investigate the basic properties of an ultrathin bilayer in detail, we placed a direct current superconducting interference device (DC-SQUID) on the ultrathin bilayer with the use of a device process. Given that the structures of qubits and SQUIDs are similar, we believe that our system will facilitate the function of reinforcing/remodeling conventional flux qubits [16].

The direct combination of a SQUID and target to produce a magnetic flux has been attempted in other multicomponent superconductors to attain a fractional vortex [18]. However, with the exception of one of our previous publications [16], to our knowledge, there is no other report on the identification of the fractional vortex. We explore herein the basic properties of this device with the use of a bilayer with a through-hole. 


\section{Experiments}

Fig. 1(a) shows that the system used in this study consists of an external copper coil and a chip with the developed ultrathin bilayer disk. The coil wire had a diameter of $0.85 \mathrm{~mm}$, and the coil had an internal diameter of $7.4 \mathrm{~mm}$. The space between the coil and chip was $\sim 4.5 \mathrm{~mm}$. This coil generated the field, which was oriented perpendicular to the surface of the chip. Fig. 1(b) shows the schematics of the chip, comprising a $\mathrm{Nb}$ coil, lines connecting the terminals, and two types of SQUID arrays; one array type is used for calibration and the other for detecting fractional flux quantum trapped in the bilayer. In the right-hand-side row of the chip, the bilayer disk was present underneath a series array of 100 SQUIDs, whereas in the left row, the bilayer disk was absent. The length of the SQUID arrays was $1.2 \mathrm{~mm}$, and the separation between the centers of the two SQUID arrays was $24 \mu \mathrm{m}$. These arrays were surrounded by a rectangular $\mathrm{Nb}$ coil, with an inner width and length of $1 \mathrm{~mm}$ and $5 \mathrm{~mm}$, respectively, and a Nb linewidth of $200 \mu \mathrm{m}$. Figs. 1 (c)-(e) show the SQUID and bilayer in detail. The disk with a diameter of $10 \mu \mathrm{m}$ consisted of two 20-nm-thick Nb layers. We inserted a 5-nm-thick Al layer between the two $\mathrm{Nb}$ layers, and the top surface of this $\mathrm{Al}$ layer was oxidized. The disk consisted of three layers. The special feature of our device is a central through-hole with a diameter of $2 \mu \mathrm{m}$ in the bilayer, which was designed with a Josephson current density of $300 \mathrm{~A} / \mathrm{cm}^{2}$ 
between the two Nb layers. The bilayer was covered with a $100-\mu \mathrm{m}$-thick silicon oxide layer, and the DC-SQUIDs (with a designed critical current of $76 \mu \mathrm{A}$ ) were placed on it. The DC-SQUID has two square junctions of dimensions $3 \mu \mathrm{m} \times 3 \mu \mathrm{m}$, and the critical current was designed to be $38 \mu \mathrm{A}$ in one junction. The series array of 100 SQUIDs improved the signal-to-noise ratio because of the averaged results of the SQUIDs. We quantified the transport properties of the SQUIDs by using a typical instrument $[16,19,20]$.

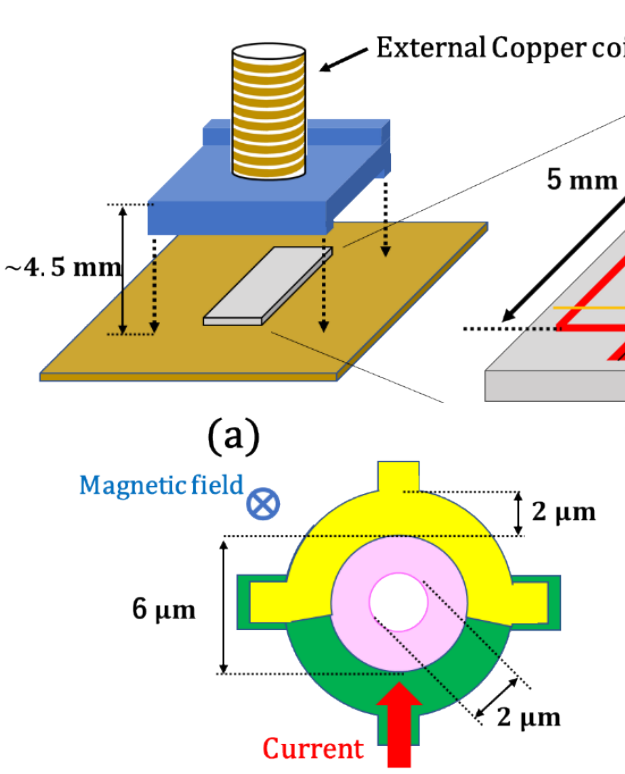

(d) (b)

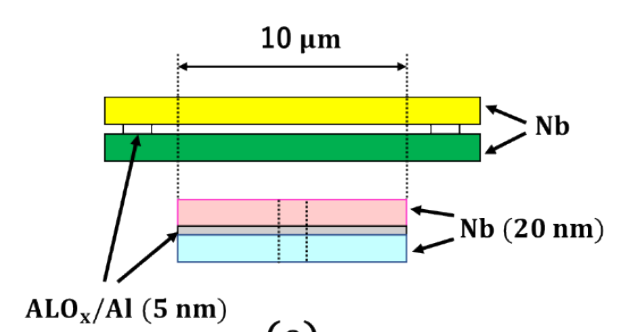

(e)

Fig. 1. Device used in experiments. Schematic of the (a) system, (b) chip, and (c) superconducting interference device (SQUID) and bilayer. (d) Top and (e) side views of the SQUID and bilayer.

We inferred that the total flux that passed through the SQUIDs was characterized by the Ic values of the SQUIDs. In the I-V characteristic, 
we determined the positive currents measured at $90 \%\left(\mathrm{IC}^{+} \quad(90 \%)\right.$ and $10 \%\left(\mathrm{IC}^{+}(10 \%)\right.$ of the maximum voltage, as well as the negative currents measured at $90 \%(\mathrm{Ic}(90 \%))$ and $10 \%(\mathrm{Ic}(10 \%))$ of the minimum voltage. When all the SQUIDs were in the same Ic state (high/low state), Ic $(10 \%) \approx I_{C}^{ \pm}(90 \%)$. In all the measurements, the SQUID bias currents were first increased from 0 to $100 \mu \mathrm{A}$, then decreased to $-100 \mu \mathrm{A}$, and finally increased back to $0 \mu \mathrm{A}$. The current flowing in the upward direction in Fig. 1(d) is the current in the positive direction.

The flux quantum was trapped as the temperature decreased and while the temperature passed through the superconducting transition point in the presence of an external field (this phenomenon is called "field cooling" (FC)), hereafter referred to as $\Phi_{\mathrm{FC}}$. The device temperature was first increased to $12-14 \mathrm{~K}$, which was higher than the critical temperature of superconductivity for the bilayer (i.e., TClayer $=7.73 \mathrm{~K}$ ); subsequently, the temperature was reduced following the application of a magnetic field. The magnetic field was then removed at temperatures in the range of 6-6.5 K. After cooling below $5.5 \mathrm{~K}$, we re-applied the field to measure the external field dependency of $\mathrm{I}_{\mathrm{c}}$ at low temperatures. The field applied at low temperatures is designated as $\Phi_{\text {ex }}$. The downward direction of the magnetic field is determined to be the positive direction. The applied magnetic field is positive when the current flow appears clockwise by looking at the chip from above.

$\Phi_{\mathrm{FC}}$ denotes the magnetic field applied at high temperatures (above 6.5 
K) when FC is generated by the external copper coil, whereas $\Phi_{\text {ex }}$ denotes the magnetic field applied at low temperatures (below $5.5 \mathrm{~K}$ ) after FC is generated by the $\mathrm{Nb}$ coil. The reasons behind the use of the two types of coils are heating and resistance. The external coil was made of copper wire, which was heated when the current was applied. When the external coil is used for a long period, it becomes difficult to maintain the coil at a constant temperature, and thus, becomes unsuitable for generating $\Phi_{\mathrm{ex}}$. In contrast, the $\mathrm{Nb}$ coil has a large resistance above $\mathrm{T}_{\mathrm{C}}^{\mathrm{Nb}}$ $=9.23 \mathrm{~K}$, and it is difficult to apply current to it at temperatures $>\mathrm{TC}^{\mathrm{Nb}}$. Thus, the $\mathrm{Nb}$ coil is unsuitable for generating $\Phi_{\mathrm{FC}}$ and operates at higher temperatures (12-14 K).

We conducted two types of calibrations for the external field. For one type of calibration, the external field was represented by the total flux inside the SQUIDs without the bilayer when the external field was applied. Subsequently, we calibrated the magnetic field with the left SQUID. The flux unit was represented by $\Phi_{0}$ in this case. For the other type of calibration, the external field was represented by the total flux inside the SQUIDs with the bilayer when the external field was applied. The flux unit was represented by $\tilde{\Phi}_{0}$ in this case. The appropriateness of the unit depends on the situation, as has been discussed later.

We investigated two types of Ic changes in the SQUIDs, which were measured at temperatures of $4-5 \mathrm{~K}$. In the $\Phi_{\mathrm{FC}}-\mathrm{IC}_{\mathrm{C}}$ characteristic 
measurements, the FC process was performed each time before the I-V characteristics were measured; the $\Phi_{\mathrm{FC}}$ value changed at every measurement. In these $\Phi_{\mathrm{FC}}-\mathrm{IC}_{\mathrm{C}}$ measurements, we maintained $\Phi_{\mathrm{ex}}=0$. In the $\Phi_{\text {ex }}-I_{c}$ characteristic measurements, the FC process was performed only once, i.e., the I-V characteristics were measured by varying $\Phi_{\mathrm{ex}}$, without increasing the device temperature again.

\section{Results and Discussion}

The I-V characteristics (Figs. 2(a) and 2(b)) depict the high and low Ic states. Typically, the SQUID voltage jumped to $\sim 280 \mathrm{mV}$, which corresponded to a value which was equal to 100 times the gap of the superconducting $\mathrm{Nb}[21,22]$. This means that all SQUIDs were in the "running state," which we were able to confirm.

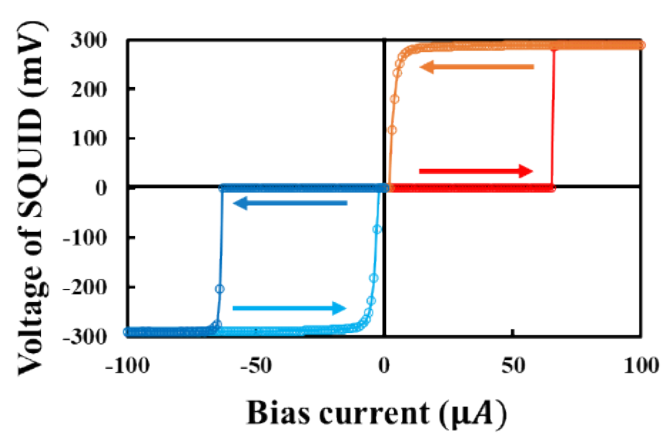

(a)

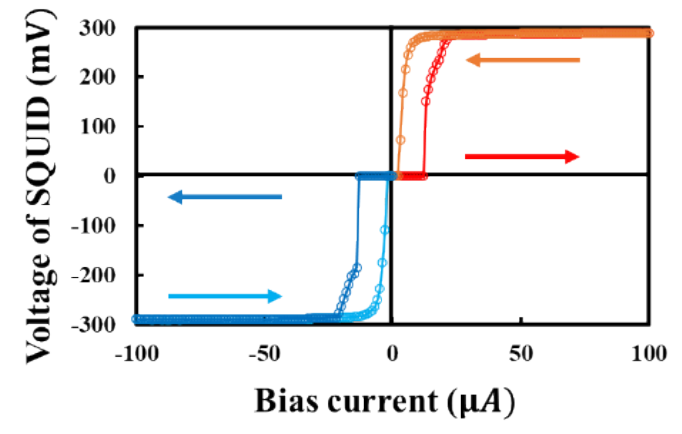

(b)

Fig. 2. I-V characteristics. Arrows indicate the directions of the currents which sweep each other. (a) Case in which $\Phi_{\mathrm{FC}}=0.0$. (b) Case in which $\Phi_{\mathrm{FC}}=1.0 \Phi_{0}$.

Figs. 3(a) and 3(b) present the $\Phi_{\text {ex }}-\mathrm{I}_{\mathrm{c}}$ characteristics for the cases of 
$\Phi_{\mathrm{FC}}=0.0$ and $\Phi_{\mathrm{FC}}=1.2 \Phi_{0}$, respectively. The hysteresis observed herein was small. A comparison of these two figures shows that the $\Phi_{\text {ex }}$ which yields the maximum $\mathrm{I}_{\mathrm{c}}$ is different in the two figures, thus indicating a shift in the signal.

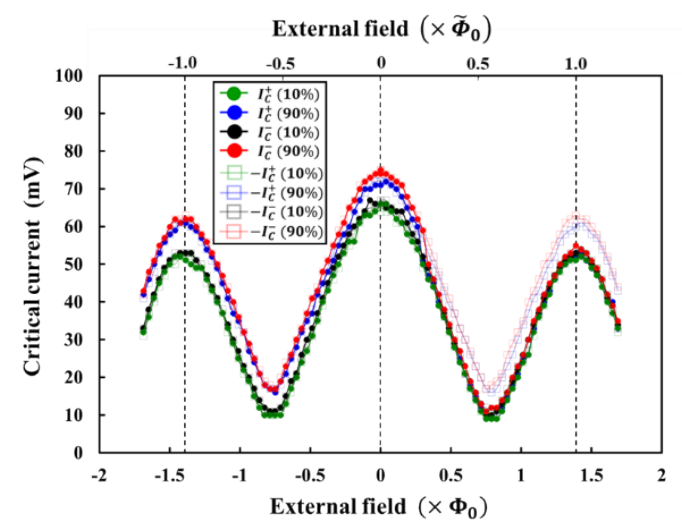

(a)

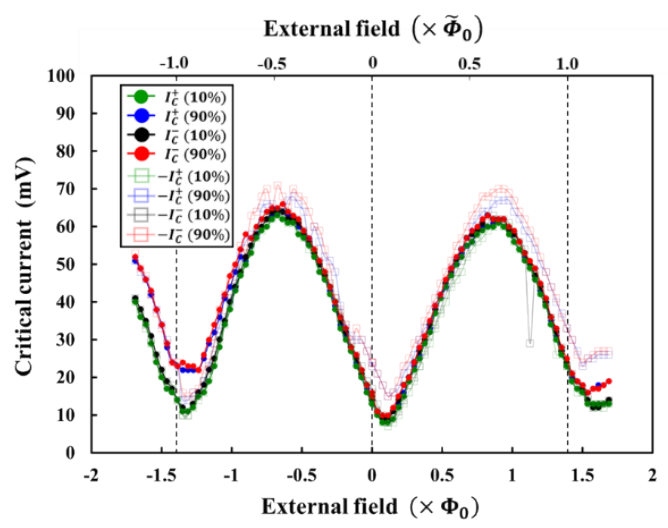

(b)

Fig. 3. $\Phi_{\text {ex }}-\mathrm{I}_{\mathrm{c}}$ characteristics: Changes in $\mathrm{I}_{\mathrm{c}}$ with respect to $\Phi_{\mathrm{ex}}$. (a) Case in which $\Phi_{\mathrm{FC}}=0.0$. (b) Case in which $\Phi_{\mathrm{FC}}=1.2 \Phi_{0}$. The dark and light lines indicate the ascending and descending trends of $\Phi_{\mathrm{ex}}$, respectively. The lower axis is scaled by $\tilde{\Phi}_{0}$, and the upper axis is scaled by $\Phi_{0}$.

$\tilde{\Phi}_{0}$ is used as the calibration value in Fig. 3. The reason behind the use of $\tilde{\Phi}_{0}$ as the calibration value in Fig. 3 is that the superconductors cancel a part of the applied field at temperatures below $T_{\mathrm{C}}$. In our system, $T_{C}^{\text {bilayer }}$ (critical temperature of the bilayer) is $7.73 \mathrm{~K}[23,24]$, and $\Phi_{\mathrm{ex}}$ is always applied below TC Tilayer $^{\text {. }} \tilde{\Phi}_{0}$ has the following relationship with $\Phi_{0}$ : $\tilde{\Phi}_{0} \approx 1.39 \Phi_{0}$. Similarly, changes in the period have been observed in the $\mathrm{Sr}_{2} \mathrm{RuO}_{4}$ - $\mathrm{Ru}$ eutectic microplates with a micro-DC-SQUID [18]. 
The Ic of the SQUID varies at $\tilde{\Phi}_{0}$ intervals with the modulation of the external magnetic fields. The value of $I_{c}$ is usually maximized at $\tilde{\Phi}_{\mathrm{ex}}=0$. The shift of the location where $I_{C}$ is maximized indicates the presence of a magnetic flux other than the external field $\tilde{\Phi}_{\text {ex }}$. In Fig. 3, this shift is equal to $0.45 \pm 0.01 \tilde{\Phi}_{0}$.

Furthermore, we investigated the value of $\Phi_{\mathrm{FC}}$ at which the phase shift occurred (Fig. 4). When the phase shift occurs, the SQUIDs should be in a low Ic state with $\Phi_{\mathrm{ex}}=0$. As is evident from Fig. 4, for current values below $0.5 \Phi_{0}$, the $\mathrm{I}_{\mathrm{C}}$ value remains high $\left(\mathrm{I}_{\mathrm{C}}=60-70 \mu \mathrm{A}\right)$; however, between $0.9 \Phi_{0}$ and $1.5 \Phi_{0}$, the $\mathrm{I}_{\mathrm{C}}$ is low $\left(\mathrm{I}_{\mathrm{C}}=15-25 \mu \mathrm{A}\right)$. Therefore, the phase shift does not occur for values below $0.5 \Phi_{0}$; instead, it occurs for values between $0.9 \Phi_{0}$ and $1.5 \Phi_{0}$. However, it should be noted that there exists a run dependence for $\Phi_{\mathrm{FC}}$ values above $1.5 \Phi_{0}$. This does not affect the confirmation of the function of the phase shifter. We are conducting extensive investigations on this aspect in an ongoing study.

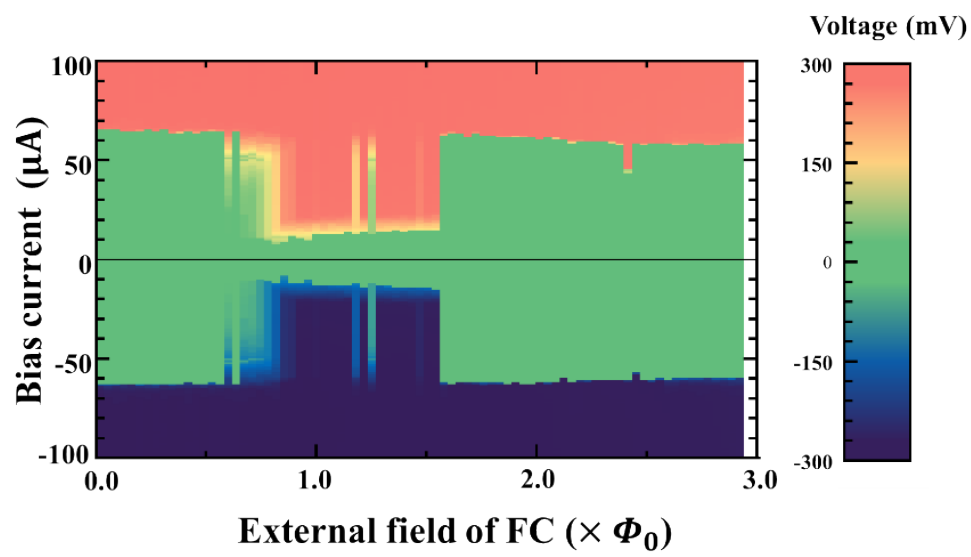

Fig. 4. $\Phi_{\mathrm{FC}}-\mathrm{l}_{\mathrm{C}}$ characteristics and color scale indicating the voltage of SQUIDs array. The boundary between yellow and red regions 
corresponds to $I_{C}^{+}(90 \%)$. The boundary between the green and yellow regions corresponds to $\mathrm{IC}_{\mathrm{C}}^{+}(10 \%)$. The boundary between the blue and green regions corresponds to $\mathrm{I} \bar{c}(10 \%)$. The boundary between indigo and blue regions corresponds to Ić (90\%).

$\Phi_{0}$ is used as the calibration value in Fig. 4. $\Phi_{0}$ yields a more appropriate value because the shielding and/or the Meissner effect of the bilayer is extremely weak when the magnetic flux enters the bilayer at temperatures just below the $-\mathrm{TC}^{\text {bilayer. }}$

Herein, we discuss the occurrence of the phase shift. We have two different interpretations to explain this phenomenon. First, a fractional flux quantum is trapped in the bilayer by the FC method. Second, a part of the flux quantum trapped in the bilayer escapes from regions between the SQUID and bilayer.

The fractional flux is generated in the ultrathin bilayer; the fractional value is determined as the ratio of the thickness of the upper to the lower layer [14, 25]. Fractional flux quanta were confirmed in an ultrathinbilayer system with a hole present only in the upper layer [14, 16]. We believe that the same phenomenon may occur in an ultrathin bilayer system with a through-hole. If a flux quantum is generated in the ultrathin bilayer system with a through-hole, the amount of shift should be $1 / 2 \Phi_{0}$ when the thickness of the upper layer is equal to the thickness of the lower layer in our system. The value of the fractional quantum can vary when there is a difference in the effective thickness between the 
lower and upper layers. Considering the proximity effect in the $\mathrm{Al}$ layer, the thicknesses of the upper and lower layers of the bilayer were slightly different $[14,16]$. The amount of shift with $0.45 \pm 0.01 \tilde{\Phi}_{0}$ can be explained by this theory.

There is space between the SQUID and the bilayer in this system. Thus, it is possible that a part of the flux trapped in the bilayer escaped through this space. To allow a part of the flux with a value of approximately 0.55 $\Phi_{0}$ to escape from regions between the SQUID and bilayer, the SQUID needs to be approximately $2000 \mathrm{~nm}$ away from the bilayer based on the assumption of a monopole-like field distribution [26-28] observed in the Pearl vortex in an infinite two-dimensional layer [6, 29]. The distance in our system is approximately in the range of $100-200 \mathrm{~nm}$; therefore, it cannot be assumed that nearly half of the flux quantum escapes.

The most plausible interpretation regarding the phase shift with 0.45 $\pm 0.01 \tilde{\Phi}_{0}$ is the first interpretation according to which the phase shift is generated by the fractional flux quanta. If our interpretation is correct, our system is ideal as a phase shifter because it can be easily designed to achieve arbitrary shifts by adjusting the thicknesses of the upper and lower layers. It does not need extra space, as observed in the flux bias given by the extra loop following the attachment of a SQUID [30]. Further, the proposed system does not require new materials, such as magnetic materials, other than $\mathrm{Nb}[31,32]$.

We confirmed that a research study has been published on a ring 
composed of a thick bilayer [33]. However, while the geometric shape of this bilayer is similar to the geometry of our ultrathin bilayer, fundamental content differences exist. In the case of the conventional bilayer, flux propagates through layers. In our ultrathin bilayer, flux goes through a pinhole in the center of the bilayer. The physical principles, properties, dynamics, basic formulas, underlying phenomena, and the role of the magnetic flux are completely different in these two cases. The "fractional phase shift" in the thick bilayer ring was also investigated [34], wherein the phase shift was induced by an externally injected current. Similar to the phase shift induced by the additional ring [30], the extra space was needed, and this system could potentially feed the noise induced by the external current lead. Using the flux trapped in the ultrathin bilayer system we can avoid these drawbacks.

\section{Conclusion}

It is worth noting that this system can be utilized as a phase shifter. We intend to investigate this phase shifting mechanism for use in a qubit. In addition, this system is useful for studying fractional flux quanta. If it can be proven that this phase shift is due to fractional flux quanta, and it can be used to assess whether fractional flux quanta are generated in multilayer systems, and multicomponent superconductors would thus become considerably simpler. 


\section{Acknowledgments}

The devices were fabricated in the clean room for analog-digital superconductivity (CRAVITY) at the National Institute of Advanced Industrial Science and Technology (AIST). We would like to thank Editage (www.editage.jp) for English language editing.

\section{Declarations}

\section{Funding}

Support was received from the AIST Innovation School.

\section{Conflicts of interest/Competing interests}

All authors declare that (i) no support, financial or otherwise, has been received from any organization that may have an interest in the submitted work, and (ii) there are no other relationships or activities that could appear to have influenced the submitted work.

\section{Availability of data and material}

The data and material that support the findings of this study are available from the corresponding author upon reasonable request.

\section{Code availability}

Not applicable. 


\section{Authors' contributions}

H. I.: original draft, data curation, formal analysis, investigation, software, visualization, review, and editing. H. Y.: conceptualization, data curation, investigation, methodology, resources, software, review, and editing. S. A.: conceptualization, review, and editing. K. T.: review and editing. Y. T.: conceptualization, original draft, data curation, formal analysis, investigation, methodology, resources, software, visualization, review, and editing.

\section{References}

1) W.E. Lowence, S. Doniach, Proc of the 12th Intern Conf. on Low Temperature Phys., Kyoto, 1970 Ed. E Kanda, Tokyo: Kigaku, $1971,361-362$.

2) G. Blatter, V. B. Geshkenbein, A. I. Larkin, Phy. Rev. Lett. 68, 875 (1992).

3) R.A. Klemm, M.R. Bealey, A. Luther, J. Low Temp. Phys. 16, 607 (1979).

4) D.E. Prober, M.R. Beasley, R.E. Schwall, Phys. Rev. B 15, 5241 
(1997).

5) J.G. Bednorz and K.A. Miller, Z. Phys. B. 64, 189 (1986).

6) J.R. Clem, Phys. Rev. B, 43, 7837 (1991).

7) Y. Kamihara, T. Watanabe, M. Hirano, H. Hosono. J. Am. Chem. Soc. 130, 3296 (2008).

8) W. Zhang and H.A. Ferting, Phys. Rev. B 71, 224514 (2005).

9) A. Gurevich and V.M. Vinokur, Phys. Rev. Lett. 97, 137003 (2006).

10) H. Bluhm, N.C. Koshnick, M.E. Huber, K.A. Moler, Phys. Rev. Lett. 97, 237002 (2006).

11) Y. Tanaka, J. Phys. Soc. Jpn. 70, 2844 (2001).

12) Y. Tanaka, Phys. Rev. Lett. 88, 017002 (2002).

13) A. Crisan, Y. Tanaka, D.D. Shivagan, A. Iyo, L. Cosereanu, K. Tokiwa, T. Watanabe, Jpn. J. Appl. Phys. 46, L451 (2007).

14) Y. Tanaka, H. Yamamori, T. Yanagisawa, T. Nishio, S. Arisawa, Physica C 548, 44 (2018).

15) S. Arisawa, K. Mochiduki, K. Yun, T. Hatano, I. Iguchi, K. Endo. 
JJAP. 51, 095804 (2021).

16) Y. Tanaka, H. Yamamori, S. Arisawa, submitted to Physica C.

17) J.E. Mooji, T.P. Orlando, L. Levitov, L. Tian, C.H. van der Wal, S. Lloyd, Science 285, 1036 (1999).

18) Y. Nago, D. Sakuma, R. Ishiguro, S. Kashiwaya, S. Nomura, K. Kono, Y. Maeno, H. Takayanagi, J. Phys. 969, 9 (2017).

19) H. Yamamori, M. Maruyama, Y. Amagai, T. Shimazaki, IEICE Electron. Express 16, 20190219 (2019).

20) A. Shoji, H. Yamamori, M. Ishizaki, S.P. Benz, P.D. Dresselhaus, IEEE TAS. 13, 919 (2003).

21) M.D. Sherrill, H.H. Edwards, Phys. Rev. Lett. 6, 460 (1961).

22) M. Hidaka, S. Nagasawa, J. Cryo. Super. Soc. Jpn. 52, 5 (2017) [in Japanese].

23) I.V. Grigorieva, W. Escoffier, J. Richardson, L.Y. Vinnikov, S. Dubonos, V. Oboznov, Phys. Rev. Lett. 96, 077005 (2006).

24) F.M. Peeters V.A. Schweigert, B.J. Baelus, P.S. Deo, Physica C. $332,(2000)$. 
25) Y. Tanaka, H. Yamamori, T. Yanagisawa, T. Nishio, S. Arisawa, Physica C 538, 12 (2017).

26) A. Al-Zoubi, F. Yang, A. Kishk, IEEE Trans. Antennas Propag. 57,789 (2009).

27) D. Guha, Y.M.M. Antar, IEEE Trans. Antennas Propag. 54, 2657 (2006).

28) I. Lim, S. Lim, IEEE Trans. Antennas Propag. 61, 5854 (2013).

29) J. Pearl, Appl. Phys. Lett. 5, 65 (1964).

30) J.B. Majer, J.R. Butcher, J.E. Mooij, Appl. Phys. Lett. 80, 3638 (2002).

31) D.S. Golubovic, W.V. Pogosov, M. Morelle, V.V. Moshchalkov. Phys. Rev. Lett. 92. 177904 (2004).

32) V.V. Ryazanov, V.A. Oboznov, A.V. Veretennikov, A. Yu. Rusanov, Phys. Rev. B 65. 020501 (2001).

33) R. Monaco, J. Mygind, V.P. Koshelets, Supercond. Sci. Technol. 33075013 (2020).

34) K. Buckenmaier, T. Gaber, M. Siegel, D. Koelle, R. Kleiner, E. 
Goldobin, Phys. Rev. Lett. 98. 117006 (2007). 